\title{
Graphics-processing-unit-accelerated simulation for longitudinal beam dynamics of arbitrary bunch trains in electron storage rings
}

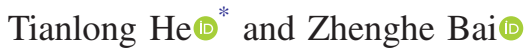 \\ National Synchrotron Radiation Laboratory, University of Science and Technology of China, \\ Hefei, Anhui 230029, China
}

(Received 6 April 2021; accepted 4 October 2021; published 15 October 2021)

\begin{abstract}
We propose a novel algorithm to deal with the interaction of beam-cavity, which is convenient to be implemented on the platform of MATLAB supporting graphics process unit operations and can significantly improve the computational efficiency. Actually, this algorithm has been implemented and the corresponding code is named STABLE, which can be used to study the longitudinal beam dynamics of arbitrary filled bunches in electron storage rings. In this paper, the STABLE code is mainly used to evaluate the bunch lengthening produced by the passive higher harmonic cavity. We first carry out efficiency tests for STABLE and the results are satisfactory. We then benchmark the STABLE code against the semianalytical approach and ELEGANT tracking for equilibrium and nonequilibrium cases, respectively, and the results are in good agreement. Furthermore, the STABLE code is applied to the case of Advanced Light Source upgrade (ALS-U) with distributed gaps, and the results firstly reveal a regular and periodic transient beam loading effect and thus show a nonequilibrium state for the overstretching case. As a second example, the STABLE code is also applied to the case of the Hefei advanced light facility (HALF) storage ring with uniformly distributed gaps, and the results show that the passive normal conducting harmonic cavities with a total $R / Q$ of $\sim 200 \Omega$ will cause severe transient effects, thus being not suitable for Hefei advanced light facility.
\end{abstract}

DOI: $10.1103 /$ PhysRevAccelBeams.24.104401

\section{INTRODUCTION}

In an electron storage ring with main parameters roughly fixed, the longitudinal beam dynamics mainly depend on the fundamental and higher order modes (HOMs) of rf cavities, trapped modes of cavity-like components, as well as the short-range wakes. Among them, the short range waying fast, only affect the single bunch beam dynamics and may cause microwave instability, and the resonant modes produce a long-lasting wakefield, which may give rise to Robinson instability or coupled bunch instability $[1,2]$. These instabilities may have an important impact on the machine's performance, and thus should be evaluated carefully.

For the fourth-generation storage ring light source, it is necessary to evaluate the influence of HOMs and the beam loading related to the fundamental mode on the beam, in order to provide a reference for the design of rf cavities, especially higher harmonic cavities (HHCs), since HHCs are widely employed for bunch lengthening to improve

\footnotetext{
"htlong@ustc.edu.cn

Published by the American Physical Society under the terms of the Creative Commons Attribution 4.0 International license. Further distribution of this work must maintain attribution to the author(s) and the published article's title, journal citation, and DOI.
}

beam lifetime and suppress intrabeam scattering. However, inappropriate design will significantly reduce bunch lengthening, cause beam instability, and ultimately limit the performance of the light source. In general, there are two types of methods that can be used for related assessments-analysis-based and tracking-based methods. For the former, there are several recently developed semianalytical methods that can calculate the equilibrium bunch profiles for arbitrary fill patterns [3-6], as well as a method that can further calculate the instability growth rate induced by HOMs on the basis of the equilibrium solution [7]. These methods have advantages that the required computing resources and calculation time are relatively small, but for some special cases, such as overstretching and nonequilibrium, they may encounter convergence problems [6]. On the contrary, the latter can be applied well to any case and can simultaneously evaluate the coupling of multiple factors but unfortunately is often limited by computing resource and calculation time. So it will be very important for tracking simulation if we can drastically reduce the requirement of the computing resource while significantly reduce the computing time, which exactly is the main motivation of this paper.

In the implementation of the longitudinal tracking algorithm, the most difficult challenge lies in the treatment of the beam-cavity interaction, especially when it comes to an arbitrary number of filled buckets. To dramatically lower 
the computational effort, the resonant wakefield action is generally represented by a voltage phasor, which is decaying, rotating and can be superimposed [8]. This voltage phasor can be updated particle by particle, so that there is no need to keep in memory the long history of previous bunch interactions. This algorithm has already been implemented into MBTRACK [9] and ELEGANT [10] and is equivalent to the so-called "wakefield matrix" employed by MUSIC [11]. It is worth noting that in MBTRACK and ELEGANT, the bunches are divided into bins (or called slices) first, and then the voltage phasor is updated bin by bin (bin-wise). In MUSIC, there is no need to obtain the bins, but the particles need to be sorted and then operated one by one (particle-wise). When dealing with the short-range wake, MBTRACK and ELEGANT need to compute directly the convolution between the bin distribution and the wake, while MUSIC needs to fit the wake into one or several resonant modes. At present, all programs mentioned above can be used to study longitudinal beam dynamics for an arbitrary number of filled buckets. However, MBTRACK and ELEGANT need large parallel computing resources which are not easily accessible, while MUSIC is nonparallel, so it often takes a long time.

In recent years, with the development of the software and hardware technology of graphics processing unit (GPU), the aforementioned large-scale computing can be carried out on a desktop personal computer through a GPU card which generally has massive processing cores. In fact, there have been several successful application cases, such as INOVESA [12], which utilizes OpenCL for parallel computation to accelerate the solution of the Vlasov-FokkerPlanck equation, and MBTRACK-CUDA [13], which employs a GPU with a CUDA programming interface and can be run on a desktop personal computer but still needs to further improve its performance, as well as the GPU-accelerated version of ELEGANT [14]. We expect to take full advantage of the GPU with a large number of processing cores and maximize its parallel computing capabilities for tracking simulation. However, whether it is the aforementioned bin by bin or particle by particle, due to the introduction of serial computing, the GPU parallel computing performance is limited to some extent.

In this paper, we will improve the algorithm for beamcavity interaction and propose a novel GPU parallel computing scheme which is very easy to be implemented. The related details will be described and the implemented code will be applied to studying the bunch lengthening for ALS-U and Hefei advanced light facility (HALF) in the presence of third-harmonic passive HHCs.

The paper is organized as follows. Section II is composed of several subsections, introduces the GPUaccelerated algorithm for the beam-cavity interaction in detail. Section II A defines the reference phase. Section II B describes the equivalent voltage phasor induced by a bunch at its reference phase. Section IIC presents the GPUaccelerated treatment for the voltage phasor induced by all previous bunch passages through rf cavities. Section II D briefly illustrates the method to deal with the short-range wake with the inclusion of the wakefield induced by the present bunch passage through rf cavities. Section III shows a simple feedback model for beam loading compensation. Section IV gives the equations of single particle motion used for the tracking implementation. Section V briefly introduces the implementation of the GPU-accelerated algorithm as well as the implemented code named STABLE. In Sec. VI, efficiency tests are conducted for STABLE and the results are satisfactory. In Sec. VII, STABLE is benchmarked against the semianalytical approach and ELEGANT and the agreement is very good. In addition, a regular and periodic transient effect is found. In Sec. VIII, the STABLE code is employed to study bunch lengthening for ALS-U and HALF and some valuable results are obtained. The conclusions are presented in Sec. IX. Appendix A derives the equivalent voltage phasor in time domain. Appendix B derives the voltage phasor for resonant modes with moderate $\mathrm{Q}$ factor. Appendix $\mathrm{C}$ explains that a reduced $\mathrm{Q}$ can be used for tracking simulation associated with a superconducting $\mathrm{HHC}$ and gives the $\mathrm{Q}$ value used for HALF.

\section{GPU-ACCELERATED ALGORITHM}

We intend to treat the voltage induced by the previous bunch passages and the present bunch passage through an rf cavity, independently, as done in Ref. [5], where the former and the latter correspond to the long-range interbunch wake interaction and the short-range intrabunch wake interaction, respectively.

\section{A. Reference phase}

In order to conveniently handle the long-range interbunch wake interaction, a reference phase is defined and attached to each rf bucket, which rotates at a fixed frequency of $h \omega_{0}$, where $h$ is the harmonic number of $\mathrm{rf}$ system and $\omega_{0}$ is the angular revolution frequency. For each bunch, the particles at the reference phase are named reference particles and have $\tau=0$, where $\tau$ is defined as the time coordinate with respect to reference particles. So the head particles have $\tau<0$ while the tail particles have $\tau>0$. Note that each rf bucket has its own reference phase and the reference phase deviation between two adjacent $\mathrm{rf}$ buckets is $2 \pi$.

\section{B. Equivalent voltage phasor}

Let us first assume that a bunch with a density distribution $\rho(\tau)$ passed through an rf cavity with quality factor $Q$, shunt impedance $R$, and angular resonant frequency $\omega_{r}$, the equivalent voltage phasor generated by this bunch at its reference phase for trailing bunches can be given by 


$$
\tilde{V}_{\mathrm{eq}}=\frac{q \omega_{r} R}{Q} \int_{-\infty}^{\infty} \rho(\tau) \exp \left[\left(-j+\frac{1}{2 Q}\right) \omega_{r} \tau\right] d \tau,
$$

where $q$ is the bunch charge and $j$ is the imaginary unit. The real part of $\tilde{V}_{\text {eq }}$ represents the real voltage seen by the trailing bunches and its positive value means energy loss. This voltage phasor will then rotate and decay with a phasor factor of $\left(j-\frac{1}{2 Q}\right)$. It is easy to know that, for a particle with $\tau_{k}$ in a trailing bunch located in the next $\mathrm{rf}$ bucket, this voltage phasor is evolved to

$$
\tilde{V}_{\mathrm{eq}} \exp \left[\left(j-\frac{1}{2 Q}\right) \omega_{r}\left(T_{b}+\tau_{k}\right)\right],
$$

where $T_{b}=T_{0} / h$, with $T_{0}$ being the revolution time.

\section{Long-range interbunch voltage phasor}

It is worth noting that Eqs. (1) and (2) were successfully applied into a semianalytical method [5]. It is later realized that they can also be applied into the tracking method for GPU-accelerated simulation. Now, let us turn to arbitrary bunch trains where each bunch is represented by a collection of macroparticles. For one of the circulating bunches, in $\mathrm{rf}$ bucket $i$, as done in Eq. (1), the equivalent voltage phasor at $\tau=0$ has the form

$$
\tilde{V}_{\mathrm{eq}, i}=\frac{q_{i} \omega_{r} R}{Q} \sum_{k=1}^{N} \exp \left[\left(-j+\frac{1}{2 Q}\right) \omega_{r} \tau_{k}\right],
$$

where $q_{i}$ is the macroparticle charge in bucket $i$ and $N$ is the total number of macroparticles. If there is no bunch in bucket $i$, then let $\tilde{V}_{\text {eq, } i}=0$. Notice that Eq. (3) can be done for all bunches in parallel.

To make the following statements more simple, $\tilde{V}_{c, i}$ and $\tilde{V}_{b, i}$ are introduced to be the total equivalent voltage phasor and the total beam loading voltage phasor at the reference phase of bucket $i$, respectively. In addition, we define $\tilde{V}_{c, 0}=\tilde{V}_{c, h}$, since the rf buckets circulate in the ring. At each turn, $\tilde{V}_{c, i}$ and $\tilde{V}_{b, i}$ can be updated by the following:

$$
\begin{gathered}
\tilde{V}_{b, i}=\tilde{V}_{c, i-1} \exp \left[\left(j-\frac{1}{2 Q}\right) \omega_{r} T_{b}\right], \\
\tilde{V}_{c, i}=\tilde{V}_{b, i}+\tilde{V}_{e q, i},
\end{gathered}
$$

where $i=1,2, \ldots, h$. We can set a initial value of $\tilde{V}_{c, 0}$ for the first bunch at the first tracking turn, which can be calculated with assuming the uniform fill pattern [15]:

$$
\tilde{V}_{c, 0}=2 F I_{0} R \cos (\psi) e^{j \psi}
$$

where $F \sim 1$ for short bunches, $I_{0}$ is the average beam current, and $\psi$ is the detuning angle determined by

$$
\tan (\psi)=2 Q \frac{\omega_{r}-n h \omega_{0}}{n h \omega_{0}},
$$

where $n$ is the harmonic order of the cavity.

If $\tilde{V}_{b, i}$ is obtained by Eqs. (4) and (5), then the total longrange interbunch voltage phasor seen by a particle with $\tau_{k}$ in bucket $i$ takes the form

$$
\tilde{V}_{b, i} \exp \left[\left(j-\frac{1}{2 Q}\right) \omega_{r} \tau_{k}\right] .
$$

For above equations, Eqs. (4) and (5) can be easily handled with a function, in which only $h$ times of complex multiplication and $h$ times of complex addition operations are required. Equations (3) and (8) are parallel in particle level, so being very suitable for parallel computing with GPU card which normally has hundreds or even thousands of processing cores. Compared with the particle-wise method as mentioned above, although the overall amount of operations in Eqs. (3) and (8) is increased, all these operations are highly parallel, which can drastically improve the calculation efficiency. Note that the above equations are obtained under the high $Q$ approximation for the fundamental mode of cavities. In order to extend them for HOMs with moderate $Q$, a similar approach can be taken, and the related details are given in Appendix B.

\section{Inclusion of the short-range wake}

For the passing bunch, Eqs. (3)-(8) are employed to deal with the total voltage phasor induced by all previous passages through the cavity, thus the voltage by the present passage is omitted by them. It is found that the influence of the present passage can be easily handled together with other kinds of short-range wakes using the bin-wake convolution method. We need to first group the particles into bins for each bunch at each tracking turn, fortunately, which can be operated in parallel in bunch level. After that, we compute the convolution of bins distribution of each bunch and the short-range wake, which can also be operated in parallel in bunch level.

\section{A SIMPLE FEEDBACK FOR BEAM LOADING COMPENSATION}

A simple feedback model is built for beam loading compensation if the cavity is operated actively, which is given by the following form

$$
\tilde{V}_{g}=\tilde{V}_{\text {set }}-\frac{1}{M} \sum_{k=1}^{M} \tilde{V}_{b, k},
$$

where $M$ is the total number of filled bunches. The generator voltage phasor is updated by the subtraction of the desired cavity voltage phasor $\tilde{V}_{\text {set }}$ and the average of all bunch beam loading phasors $\tilde{V}_{b, k}$ at their own reference 
phases. Although this model is not realistic, it can still be effectively used to make the cavity voltage stable during simulation.

\section{EQUATIONS OF SINGLE PARTICLE MOTION}

We will first apply this algorithm to the bunch lengthening study in the presence of passive HHCs, as they are used in many machines, especially diffraction-limited storage rings. With the inclusion of main-cavity (MC) and HHC beam loading, and a simple feedback for beam loading compensation of $\mathrm{MC}$, the single-particle equations of motion can be given by [9]

$$
\begin{gathered}
\frac{d \tau}{d t}=\alpha_{c} \delta, \\
\frac{d \delta}{d t}=\frac{e}{E_{0} T_{0}}\left(V_{g} \sin \left(\omega_{r f} \tau+\varphi_{g}\right)-\operatorname{Re}\left[\tilde{V}_{b, t}(\tau)\right]-\frac{U_{0}}{e}\right) \\
+\frac{q e}{E_{0} T_{0}} \int_{-\infty}^{\infty} \rho\left(\tau^{\prime}\right) W\left(\tau-\tau^{\prime}\right) d \tau^{\prime},
\end{gathered}
$$

where $\alpha_{c}$ is the momentum compaction factor, $\delta$ is the relative momentum deviation, $e$ is the electron charge, $E_{0}$ is the nominal beam energy, $\omega_{r f}$ is the angular rf frequency, $V_{g}$ and $\varphi_{g}$ are the generator voltage amplitude and phase, respectively, which are determined by Eq. (9) at each turn, $\tilde{V}_{b, t}$ is the total beam loading voltage phasor, which is calculated with Eqs. (3)-(8), $U_{0}$ is the total energy loss per turn with the inclusion of the insertion devices (IDs) and $W(\tau)$ is the total short-range wake.

For electron storage rings with strong synchrotron radiation, the radiation damping and quantum excitation should be considered. Then at each turn, the coordinate $\delta$ of a particle can be updated by [9]

$$
\delta=\delta\left(1-\frac{2 T_{0}}{\tau_{z}}\right)+2 \sigma_{\delta_{0}} \sqrt{\frac{T_{0}}{\tau_{z}}} \delta_{\text {rand }},
$$

where $\tau_{z}$ is the longitudinal damping time, $\sigma_{\delta_{0}}$ is the natural energy spread, and $\delta_{\text {rand }}$ is a random number drawn from a normal distribution with unit standard deviation.

\section{IMPLEMENTATION}

The GPU-accelerated algorithm has been implemented into a MATLAB-based program, where the macroparticle coordinates of all bunches are organized into two matrices with $N$ rows and $M$ columns, each matrix stores one dimensional coordinate and each column in a matrix represents one bunch. During the implementation, except that one function to handle Eqs. (4) and (5) and another function to handle "grouping into bins" are needed to be specially programmed, all other functions already exist and can be taken directly from the GPU function library supported by MATLAB. It means that we do not need make large efforts to complete this implementation.

This code was initially developed to be a simulation tool for arbitrary filled bunch lengthening study in electron storage rings, which is abbreviated as STABLE. At present, STABLE can be conveniently used for arbitrary bunch trains and arbitrary charge configurations, which can be run on a desktop personal computer with a consumer-grade GPU card.

\section{EFFICIENCY TEST}

With a GPU card-GEFORCE GTX 1650, which has 896 CUDA cores, we test the code with the examples of ALS-U and HALF. The parameters used for ALS-U are taken from Refs. $[4,6]$, and the parameters used for HALF are listed in Table I, where the radiation loss from IDs are considered, as well as the beam loading of MC. The specific parameters of the rf system of HALF are still under design, so the parameters presented here (assuming that both the MC and passive HHC are superconducting) are only used for theoretical calculation and analysis.

The fill pattern with uniformly distributed gaps is considered for HALF, which can be simply represented by $20 \times(36 b+4 e)$. It means 20 equal subtrains spaced by 4 empty buckets and each subtrain has 36 bunches. In order to accelerate the convergence of results, a lower HHC's $Q$ of $2 \times 10^{5}$ is used rather than that listed in Table I, and the corresponding detuning of HHC is set to $66.813 \mathrm{kHz}$. With the distributed gaps, 284 and 720 bunches are tracked for ALS-U and HALF, respectively, the macroparticle number per bunch is set to $1 \times 10^{3}, 5 \times 10^{3}$, and $1 \times 10^{4}$, respectively, and the number of tracking turns is set to $2 \times 10^{4}$. The results of the calculation time are listed in Table II.

TABLE I. Main parameters of $\operatorname{HALF}(R / Q$ value is given in circuit definition).

\begin{tabular}{lcc}
\hline \hline Parameter & Symbol & Value \\
\hline Ring circumference & $C$ & $480 \mathrm{~m}$ \\
Beam energy & $E_{0}$ & $2.2 \mathrm{GeV}$ \\
Average beam current & $I_{0}$ & $350 \mathrm{~mA}$ \\
Longitudinal damping time & $\tau_{z}$ & $12 \mathrm{~ms}$ \\
Momentum compaction & $\alpha_{c}$ & $6.3 \times 10^{-5}$ \\
Natural energy spread & $\sigma_{\delta}$ & $6.6 \times 10^{-4}$ \\
Harmonic number & $h$ & 800 \\
Energy loss per turn (with IDs) & $U_{0}$ & $600 \mathrm{keV}$ \\
Voltage of MC & $V_{r f}$ & $1.235 \mathrm{MV}$ \\
R/Q of MC & $(R / Q)_{m}$ & $90 \Omega$ \\
Loaded quality factor of MC & $Q_{m}$ & $4.035 \times 10^{4}$ \\
Detuning of MC & $\Delta f_{m}$ & $-16 \mathrm{kHz}$ \\
Load angle of MC & $\theta_{L}$ & $-21 \mathrm{deg}$ \\
Harmonic order of HHC & $n$ & 3 \\
R/Q of HHC & $(R / Q)_{h}$ & $45 \Omega$ \\
Quality factor of HHC & $Q_{h}$ & $2 \times 10^{8}$ \\
Detuning of HHC & $\Delta f_{h}$ & $66.918 \mathrm{kHz}$ \\
\hline \hline
\end{tabular}


TABLE II. Calculation time for ALS-U and HALF with the fill pattern of distributed gaps and $2 \times 10^{4}$ tracking turns.

\begin{tabular}{lccc}
\hline \hline Machine & Bunch number & Particles per bunch & Time $[\mathrm{s}]$ \\
\hline ALS-U & 284 & $1 \times 10^{3}$ & 79 \\
ALS-U & 284 & $5 \times 10^{3}$ & 154 \\
ALS-U & 284 & $1 \times 10^{4}$ & 249 \\
HALF & 720 & $1 \times 10^{3}$ & 108 \\
HALF & 720 & $5 \times 10^{3}$ & 295 \\
HALF & 720 & $1 \times 10^{4}$ & 529 \\
\hline \hline
\end{tabular}

It is evident that the calculation time for ALS-U with $1 \times 10^{4}$ particles per bunch is about $4 \mathrm{~min}$, which is comparable to or even faster than that calculated by semianalytical methods $[3,6]$. The calculation for HALF with 720 bunches takes about 9 min, compared with ALS-U which has 284 bunches, the bunch number is increased by a factor of 2.5 , but the calculation time is only increased by a factor of 2.1. With increase of particle number from $1 \times 10^{3}$ to $1 \times 10^{4}$ by a factor of 10 , while the calculation time is only increased by a factor of 3.15 and 4.9 for ALS-U and HALF, respectively. The calculations for both ALS-U and HALF are expected to be even faster when a GPU card with more CUDA cores is employed. It is gratifying that the latest GPU card has more than 10 thousand CUDA cores which increased more a factor of 10 than the present one. If we only care about the bunch length and bunch centroid position, and do not care about the specific bunch profile, it seems that $1 \times 10^{3}$ macroparticles per bunch are enough to get better results, as shown in Fig. 1. For this case, the whole tracking takes only $79 \mathrm{~s}$ for ALS-U and $108 \mathrm{~s}$ for HALF, which is definitely satisfactory.
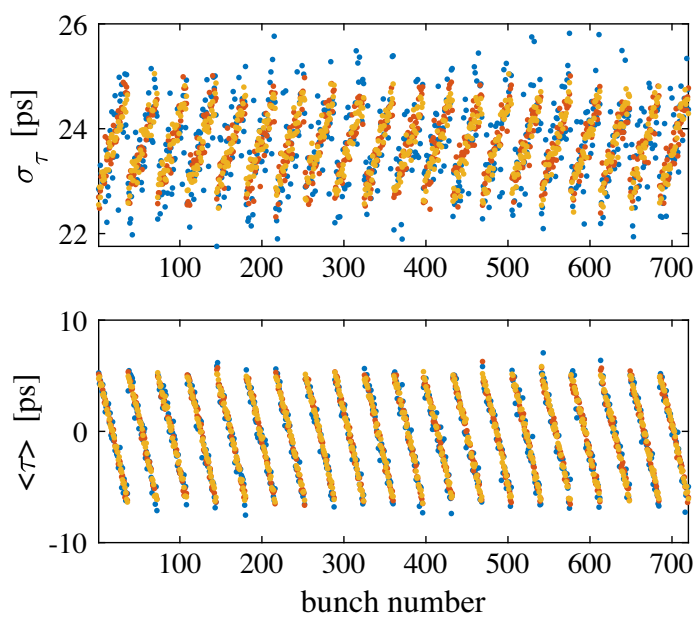

FIG. 1. RMS lengths (top) and centroid positions (bottom) vs bunch number for HALF with 720 bunches in filling case of uniformly distributed gaps, the blue, red, and yellow dots are corresponding to 1000,5000 , and 10000 particles per bunch, respectively. Fill pattern: $20 \times(36 b+4 e)$.

\section{BENCHMARK TEST}

The STABLE code can be benchmarked against two other methods. One is the semianalytical method [5], which was previously described and has recently been extended to include the beam loading of the fundamental cavity with a simple feedback scheme described in Sec. III. The other is the tracking method used in ELEGANT, which can be accelerated with the parallel version (PELEGANT) on a large computing cluster. The former will be used to check the results in the equilibrium state, while the latter will be used for the nonequilibrium case.

\section{A. Uniformly distributed gaps}

The fill pattern with uniformly distributed gaps will be preferred by the HALF storage ring, as it can effectively mitigate the influence of the transient beam loading on bunch lengthening and ensure that the bunches are stretched by a factor of 4 [5].

Here, we consider another fill pattern with uniformly distributed gaps, which is given by $16 \times(45 b+5 e)$. The parameters of HHC which are different from those listed in Table I are given by $Q_{h}=1 \times 10^{5}$ and $\Delta f_{h}=66.497 \mathrm{kHz}$. Note that, a lower $Q$ is used to speed up the convergence of tracking results, which might cause a minor difference from the correct results, as seen in Appendix C. Besides, to guarantee good bunch profiles, the number of macroparticles per bunch and tracking turns are set to $1 \times 10^{4}$ and $1 \times 10^{5}$, respectively.

Using the semianalytical method and the STABLE code, the rms lengths and centroid positions of all bunches are calculated and illustrated in Fig. 2, and the bunch profiles of three representative bunches in the first subtrain are shown in Fig. 3. It is clear that the results by the two methods are in good agreement.
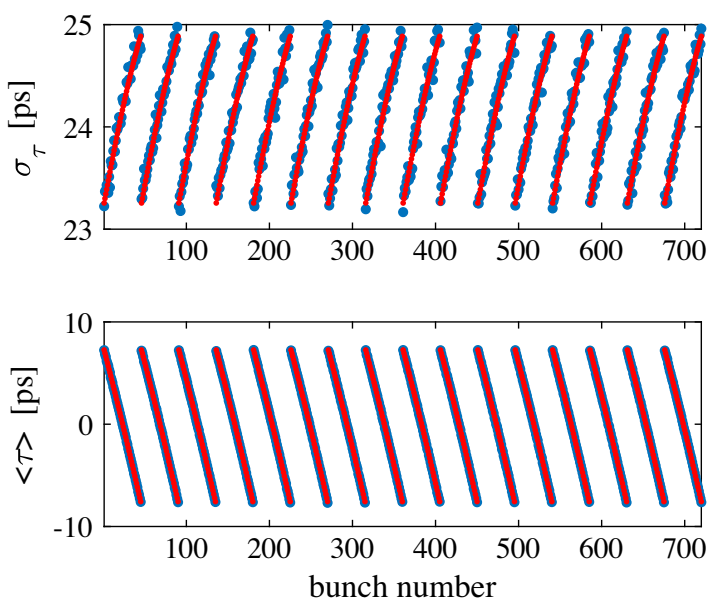

FIG. 2. RMS lengths (top) and centroid positions (bottom) vs bunch number for HALF with 720 bunches in the filling case of uniformly distributed gaps, obtained by the semianalytical method (red dots) and the tracking method (blue dots). Fill pattern: $16 \times(45 b+5 e)$. 


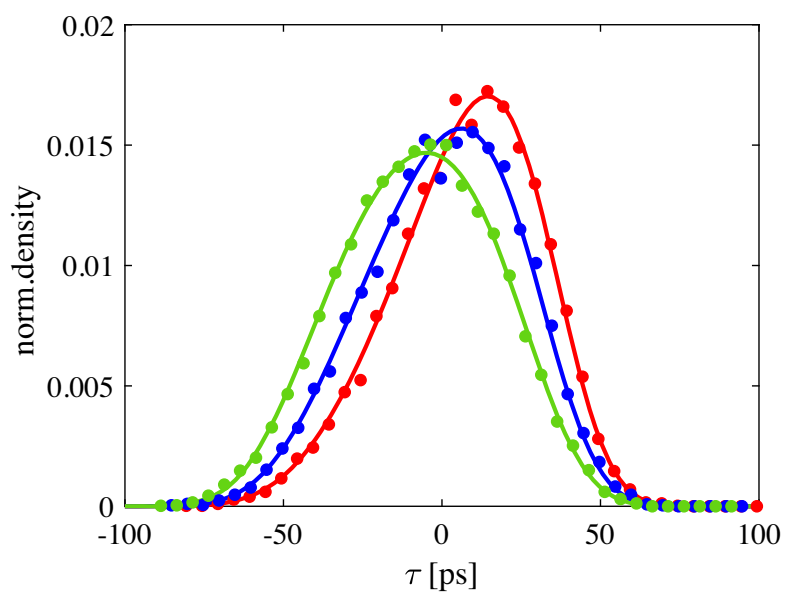

FIG. 3. Bunch profiles, 1st (red), 23rd (yellow), and 45th (blue) bunches are presented. The results of the curves and dots are given by the semianalytical and tracking methods, respectively.

\section{B. Uniform fill with 80 bunches}

For convenience, the beam loading of $\mathrm{MC}$ is not taken into account and an uniform fill pattern with 80 bunches is taken as a benchmarking example. The fill pattern is set to $80 \times(1 b+9 e)$, which may not be employed for the HALF storage ring but can reduce the computational load significantly. In order to create a nonequilibrium case, the parameters of $\mathrm{HHC}$ are set to $(R / Q)_{h}=100 \Omega, Q_{h}=$ $21 \times 10^{3}$, and $\Delta f_{h}=144.36 \mathrm{kHz}$. This HHC can be a normal conducting cavity operated in passive mode.

The variation of centroid positions and rms lengths over tracking turns are displayed in Fig. 4. In general, the results obtained by both codes tend to be consistent and can tell us the same things: 1 . Bunches cannot reach equilibrium states. Their rms lengths vary in the range of 11 to $74 \mathrm{ps}$. Besides, their centroids change very slowly from 80 to $-60 \mathrm{ps}$ (taking about $8 \times 10^{6}$ turns), and relatively fast from -60 to $80 \mathrm{ps}$ (taking less than $2 \times 10^{5}$ turns). On the whole, the states of all bunches go through the same cycle with the same periods of about 13.8 and $14.0 \mathrm{~s}$ obtained by STABLE and PELEGANT, respectively, which reflecting a kind of periodic instability. 2. Bunches are not finally uniform even though the fill pattern is uniform. In other words, the transient beam loading effect occurs even when the beam is uniformly filled.
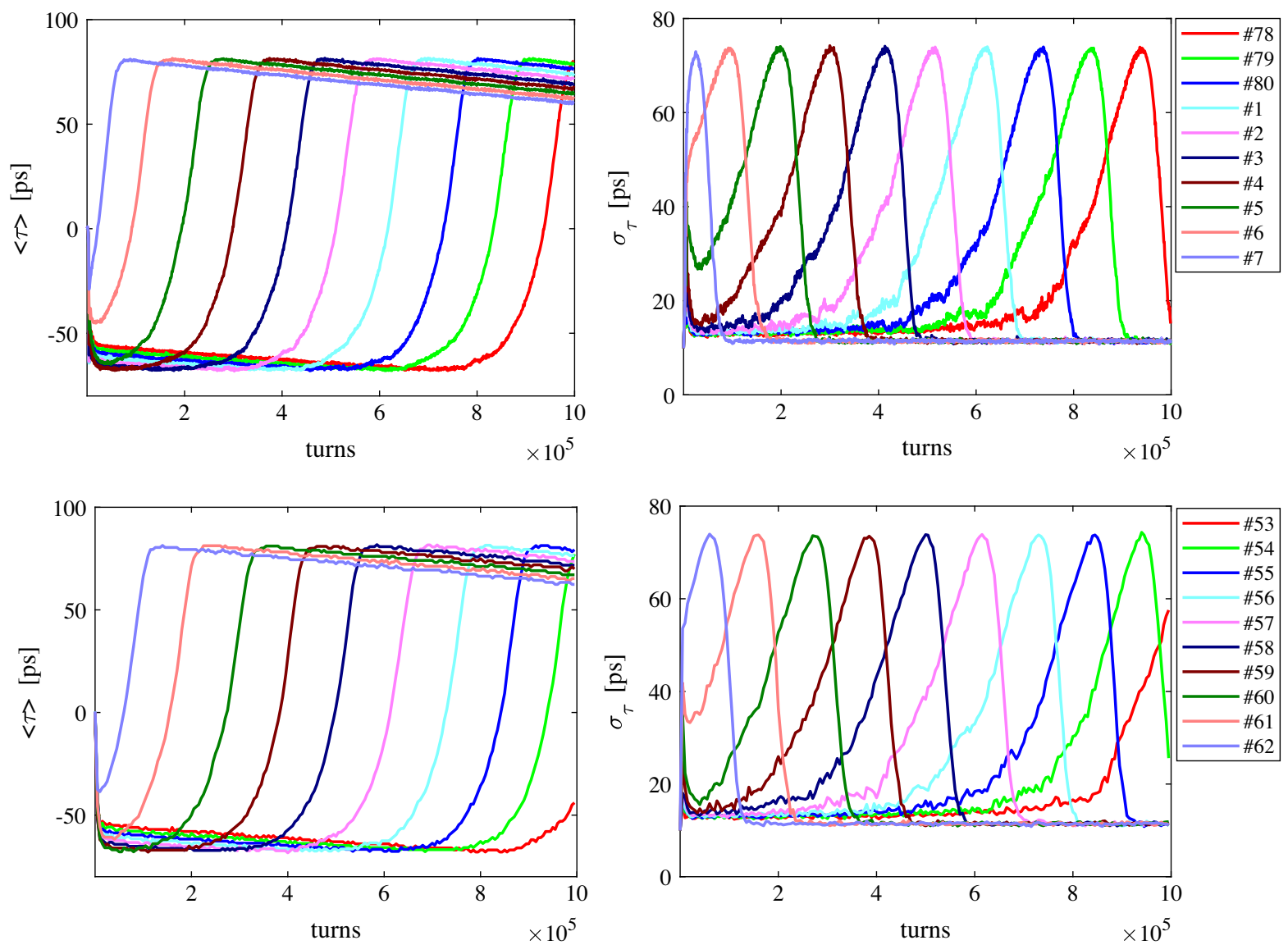

FIG. 4. Centroid positions (left) and rms lengths (right) vs tracking turns, by STABLE (top) and PELEGANT (bottom) with 2000 macroparticles per bunch and $1 \times 10^{6}$ tracking turns. Ten representative bunches are displayed and distinguished by different colors. The PELEGANT code with 80 CPU cores takes about $935 \mathrm{~min}$ to complete the calculation, while the STABLE code with 896 CUDA cores only takes about $43 \mathrm{~min}$. 
However, we also notice that there are differences between the two results. These differences can be explained by the fact that both the generation of initial coordinates of macroparticles and the treatment of quantum excitation process at each turn are randomly handled by different random generators.

\section{APPLICATION}

\section{A. ALS-U: Distributed gaps}

The STABLE code is first applied to studying the bunch lengthening for ALS-U. The fill pattern with distributed gaps is the same as in Ref. [6], and the overstretching case is checked by the STABLE code as it was reported that Warnock's method could only converge to the beam current of $474.5 \mathrm{~mA}$. We first study the case of $474.5 \mathrm{~mA}$ with the damping time of $12 \mathrm{~ms}, 4 \times 10^{3}$ macroparticles per bunch and $1 \times 10^{5}$ tracking turns. The results of centroid positions are presented in Fig. 5. It is evident that a coupled bunch instability with $l=0$ mode occurs, which grows slowly and can be theoretically suppressed by reducing the damping time. So we set the damping time down to $6 \mathrm{~ms}$, and then all bunches can reach a steady state as expected. The results of rms lengths and centroid positions are shown in Fig. 6. In addition, this case is also calculated using a semianalytical method [5], and the results are also shown in Fig. 6. We can see that both results are in good agreement. Due to the slightly different generator voltages used, the results shown in Fig. 6 have a slight difference from those in Ref. [6].

Then, we set the beam current to $480 \mathrm{~mA}$ without changing other parameters and the corresponding results are shown in Fig. 7. We can clearly see that the centroid positions and rms lengths vary with the tracking turns, which means the beam can not reach an equilibrium state. However, on the whole, their motion is regular, periodic, and oscillates much more slowly compared to the case in

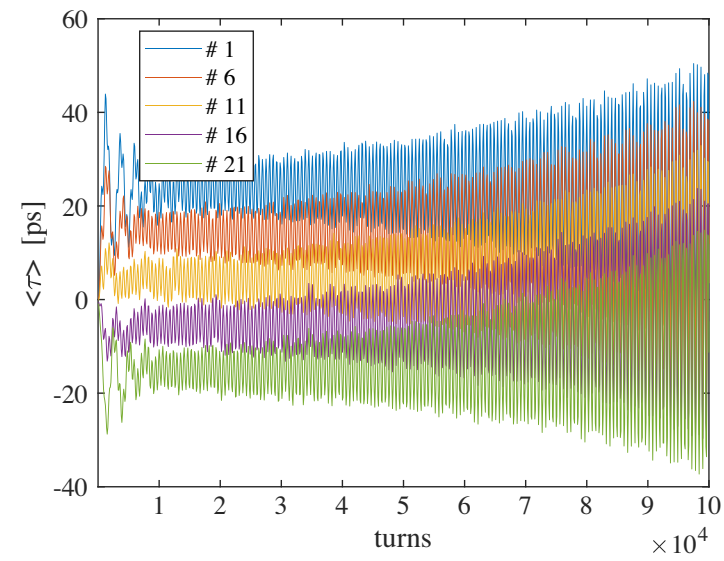

FIG. 5. Centroid positions vs tracking turns, by STABLE with $1 \times 10^{5}$ turns. Five representative bunches in the first subtrain are displayed, and distinguished by different colors. coupled bunch instabilities. We also try to set the damping time down to $2 \mathrm{~ms}$ or even $0.2 \mathrm{~ms}$, and still find the periodic phenomenon. It illustrates that this instability cannot be suppressed by reducing the damping time, which is obviously different from the general coupled bunch instability. For this overstretching case, the tracking results can directly show one very likely reason why no convergent results can be obtained in Ref. [6]. That is to say, when the beam current exceeds $474.5 \mathrm{~mA}$, all bunches cannot reach a steady state, but fall into a regular and periodic dynamic state.

More details about this periodic instability are shown in Fig. 8. First of all, the left plot shows the variation of the centroid positions along the bunch number, it is clear that the centroid position in each subtrain decreases linearly with the bunch number. Unlike the equilibrium case shown in Fig. 6, the centroid difference between two different subtrains increases significantly. If the bunches in each subtrain are regarded as a whole, the centroid position arrangement among the subtrains shows a longitudinal coupling with $l=1$ mode. Then, the right plot displays the variation of centroid positions in the first subtrain from the 50,000th to the 100,000th turn. It can be seen that if the fast synchrotron oscillation is ignored, these curves are roughly parallel, indicating that the centroid positions of all bunches in the same subtrain vary synchronously. This synchronous variation indicates that it is reasonable to regard each subtrain as one whole while talking about the coupling relationship between bunches.

The tracking results reveal the coupled motion with mode $l=1$ among subtrains, but it should be pointed out that this coupling is different from the general coupled bunch instability. Because the general coupled bunch instability involves the concepts of frequency shift and growth rate, so it can be suppressed when the damping rate
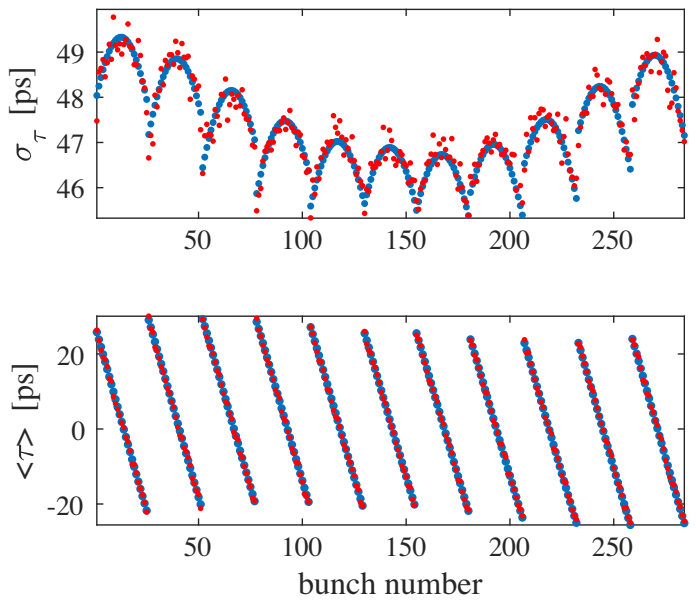

FIG. 6. RMS lengths (top) and centroid positions (bottom) vs bunch number for ALS-U with 284 bunches in the filling case of distributed gaps, obtained by the semianalytical method (blue dots) and STABLE (red dots). The damping time is set to $6 \mathrm{~ms}$ for tracking simulation. 

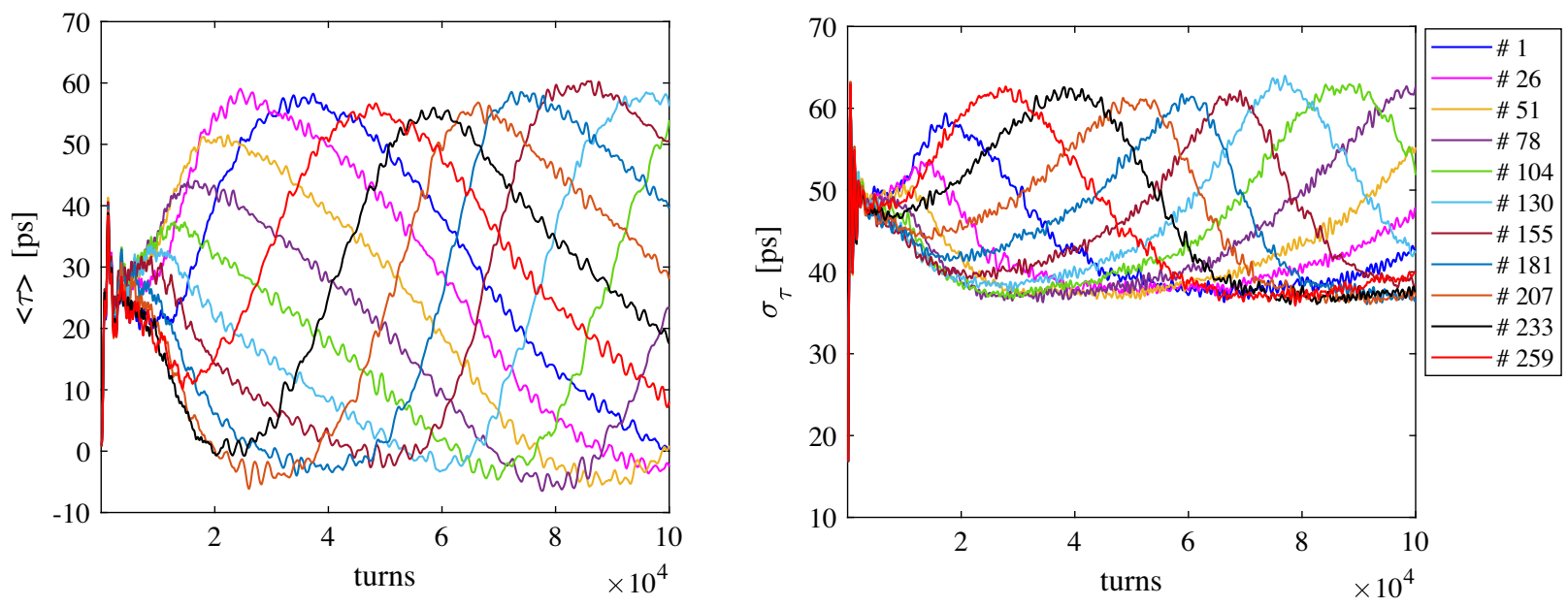

FIG. 7. Centroid positions (left) and rms lengths (right) vs tracking turns, by STABLE with $1 \times 10^{5}$ turns. The damping time is set to $12 \mathrm{~ms}$. The head bunches in all 11 subtrains are shown, and distinguished by colors.
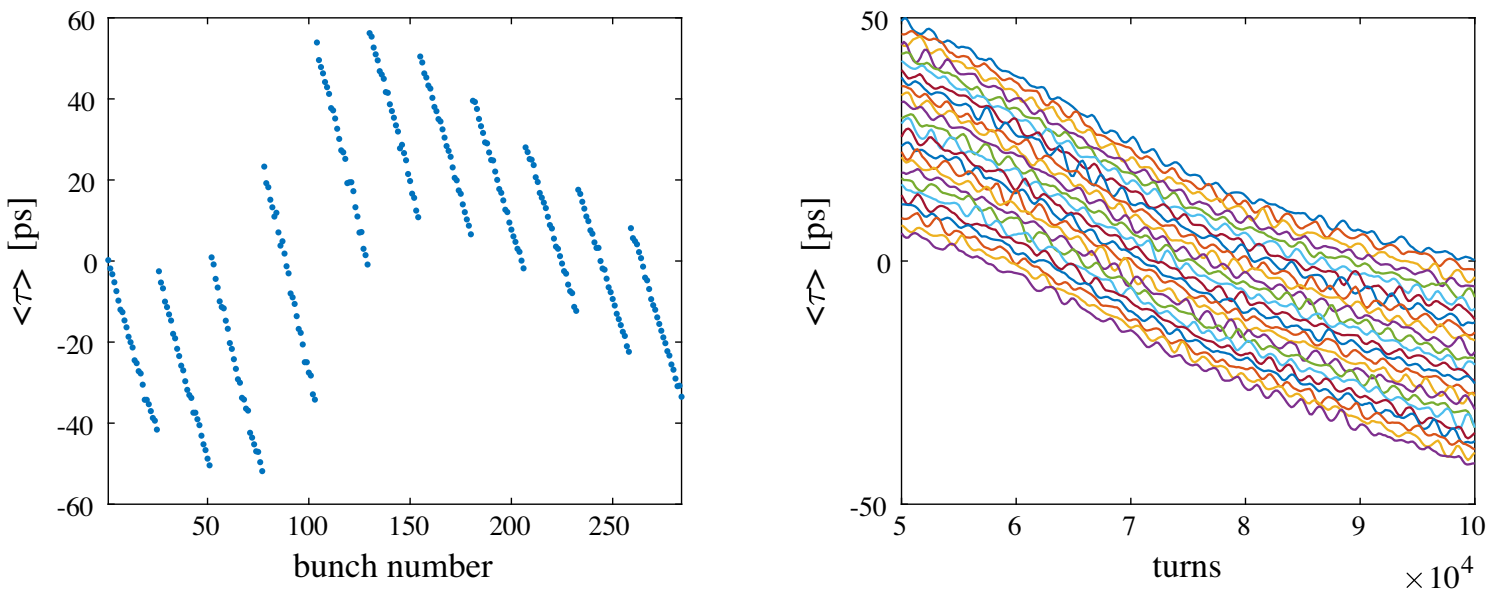

FIG. 8. Centroid positions vs bunch number (left) and tracking turns (right). The left plot shows the results at the 100,000th tracking turn. The right plot presents the results of 25 bunches in the first subtrain.

is higher than the growth rate. But it is evident that when we set a shorter damping time, the periodic instability cannot be suppressed.

We also found that, for this overstretching case, the periodic instability can be avoided and the equilibrium results can be obtained with setting a lower $R / Q$ or a higher MC voltage, which are usually adopted to reduce the transient beam loading. Therefore, we tend to consider this kind of instability as a periodic transient beam loading effect. A reasonable qualitative explanation for this periodic instability, from the tracking point of view in time domain, is that the variation of bunch centroids and beam loading voltage phasors will drive each other in turn. In normal cases, it will converge to an equilibrium state. But in some specific cases, for example, the cases where the fill pattern is proper (uniform or distributed gaps) and the value of $R / Q$ or beam current is high, it will tend to a periodic nonequilibrium state. It is necessary to further explore the dependence of this periodic instability on $R / Q$, MC voltage, fill pattern, etc., but this, however, is beyond the scope of this paper. Further study on this periodic instability will be conducted in the near future.

\section{B. HALF: Uniformly distributed gaps}

In Sec. VII, we obtained good and stable results for $R / Q=45 \Omega$ but regular and periodic results for $R / Q=100 \Omega$. It hints that the value of $R / Q$ might play an import role in bunch lengthening and stability. We need to evaluate the influence of the value of $R / Q$ on the beam bunch by considering a more practical situation for the HALF storage ring. There are two types of passive harmonic cavities-superconducting and normal conducting. Ignoring the technical difference, from the beam point of view, the main differences between the two cavities are the value of $R / Q$ and detuning frequency. Considering 
TABLE III. Parameters of four cases used for calculation.

\begin{tabular}{lrrrll}
\hline \hline & $(R / Q)_{h}[\Omega]$ & \multicolumn{1}{c}{$Q_{h}$} & \multicolumn{1}{c}{$\Delta f_{h}[\mathrm{kHz}]$} & $V_{r f}[\mathrm{MV}]$ & $\Delta f_{m}[\mathrm{kHz}]$ \\
\hline Case 1 & 90 & $5 \times 10^{5}$ & 133.83 & 1.235 & -15.7 \\
Case 2 & 90 & $5 \times 10^{5}$ & 104.28 & 1.5 & -13.4 \\
Case 3 & 200 & $21 \times 10^{3}$ & 295.26 & 1.235 & -16.0 \\
Case 4 & 200 & $21 \times 10^{3}$ & 183.52 & 1.8 & -11.5 \\
\hline \hline
\end{tabular}

the requirement of cavity voltage and the limitation of power, one superconducting cavity (like Super-3HC of SLS/Elettra [16]) or at least three normal conducting cavities (like ALS type) can meet the requirements of HALF. The total $R / Q$ can reach about $90 \Omega$ for the former, while about $200 \Omega$ for the latter. In the following calculations, the detuning of $\mathrm{HHC}$ is determined by the nearoptimum lengthening condition [5] and the detuning of $\mathrm{MC}$ is set to make the loading angle to be about -20 deg [17]; the fill pattern is set to $20 \times(36 b+4 e)$ and the number of macroparticles per bunch and the number of tracking turns are set to $2 \times 10^{3}$ and $1 \times 10^{6}$, respectively. We will present two superconducting cases and two normal conducting cases. The related parameters of cavities used for calculations are summarized in Table III. It should be noted that, for superconducting cases 1 and 2, the $Q$ factor of $5 \times 10^{5}$ rather than a real value of $\sim 1 \times 10^{8}$ is taken to significantly reduce the number of tracking turns required for result convergence, which can be done as it hardly affects the correct results, as discussed in Appendix C.

Now, we first discuss the superconducting cases 1 and 2 . Figure 9 shows the corresponding variation of centroid positions and rms lengths with the bunch number, which are obtained by STABLE at the end of tracking and semianalytical calculation. For case 1, both the tracking and semianalytical results are finally convergent which means all bunches can reach equilibrium states. However, in the same fill pattern, the convergent results here are obviously different from those shown in Fig. 1. We can see that (1) the maximum centroid difference among bunches is more than $110 \mathrm{ps}$ and (2) several bunches have rms lengths more than $40 \mathrm{ps}$, which means they are overstretched, while most bunches have rms lengths ranged from 12 to 20 ps. It should be noted that the results obtained by the two methods look different but have almost the same shape and can be coincided with each other by shifting either of the two, and the shifting number is exactly equal to the number of bunches in one subtrain. Actually, both results are equivalent, since the subtrains are equally spaced and have equal number of bunches. For case 2 with a higher $\mathrm{MC}$ voltage of $1.5 \mathrm{MV}$, the tracking results of different subtrains are not only convergent but also consistent, which are also in a good agreement with the semianalytical results. It indicates that the severe centroid transient in case 1 can be completely suppressed by increasing the MC voltage.
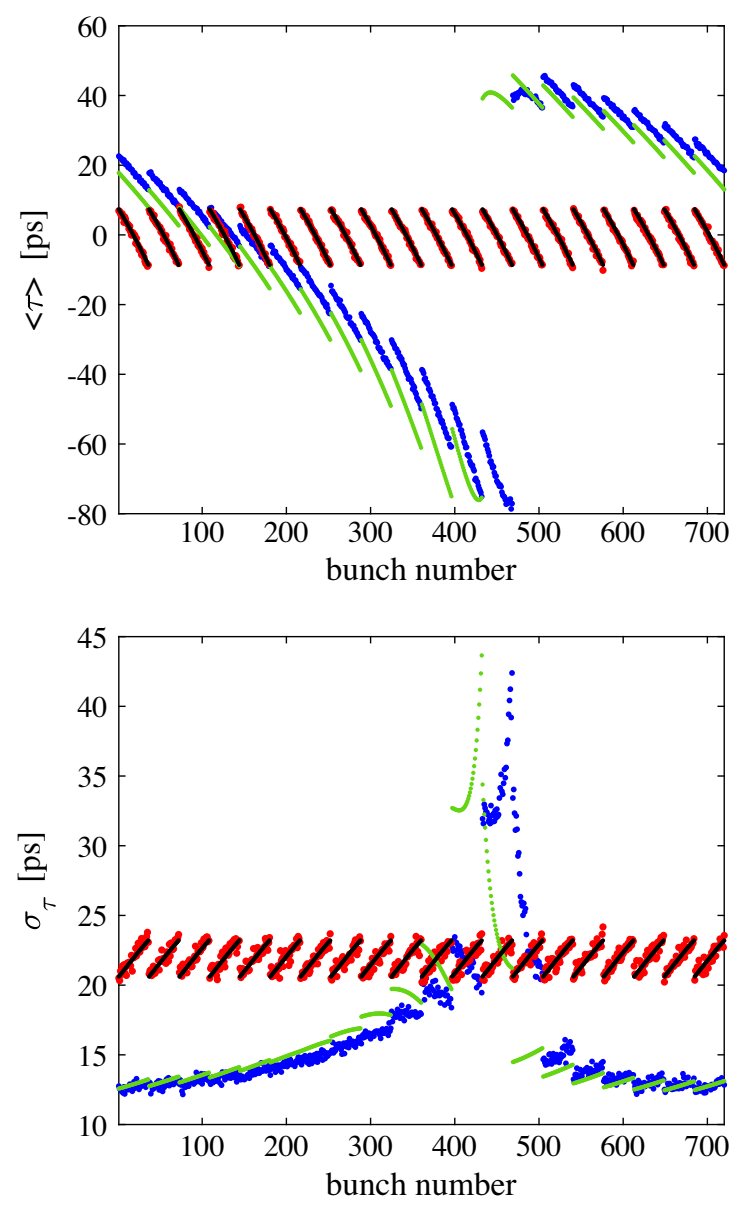

FIG. 9. Centroid positions (top) and rms lengths (bottom) vs bunch number, given by STABLE (blue dots for case 1 and red dots for case 2) and a semianalytical method (green dots for case 1 and black dots for case 2).

It is worth noting that it is generally believed that the results should be periodically distributed as the fill pattern has periodically distributed bunches. However, the results of case 1 shown in Fig. 9 are obviously different from what we generally think. How to understand this abnormal phenomenon? A possible explanation is that this phenomenon is mainly determined by the $\mathrm{MC}$ voltage amplitude as well as the beam loading strength of HHC which increases with the $R / Q$ and beam current. When the beam loading strength exceeds a certain threshold, the original stable distribution becomes disturbed and gradually forms a periodic motion state and leads to the centroid difference between neighboring subtrains. When the beam loading is strong enough, the difference between one pair of adjacent subtrains becomes large enough, which will lead to the truncation of the periodic motion and the convergence of an equilibrium state. When the MC voltage increases, the beam loading strength will become relatively weakened and the result can thus return a periodic stable distribution.

Next, we will discuss the normal conducting cases 3 and 4 . Figure 10 shows the variation of centroid positions and rms 

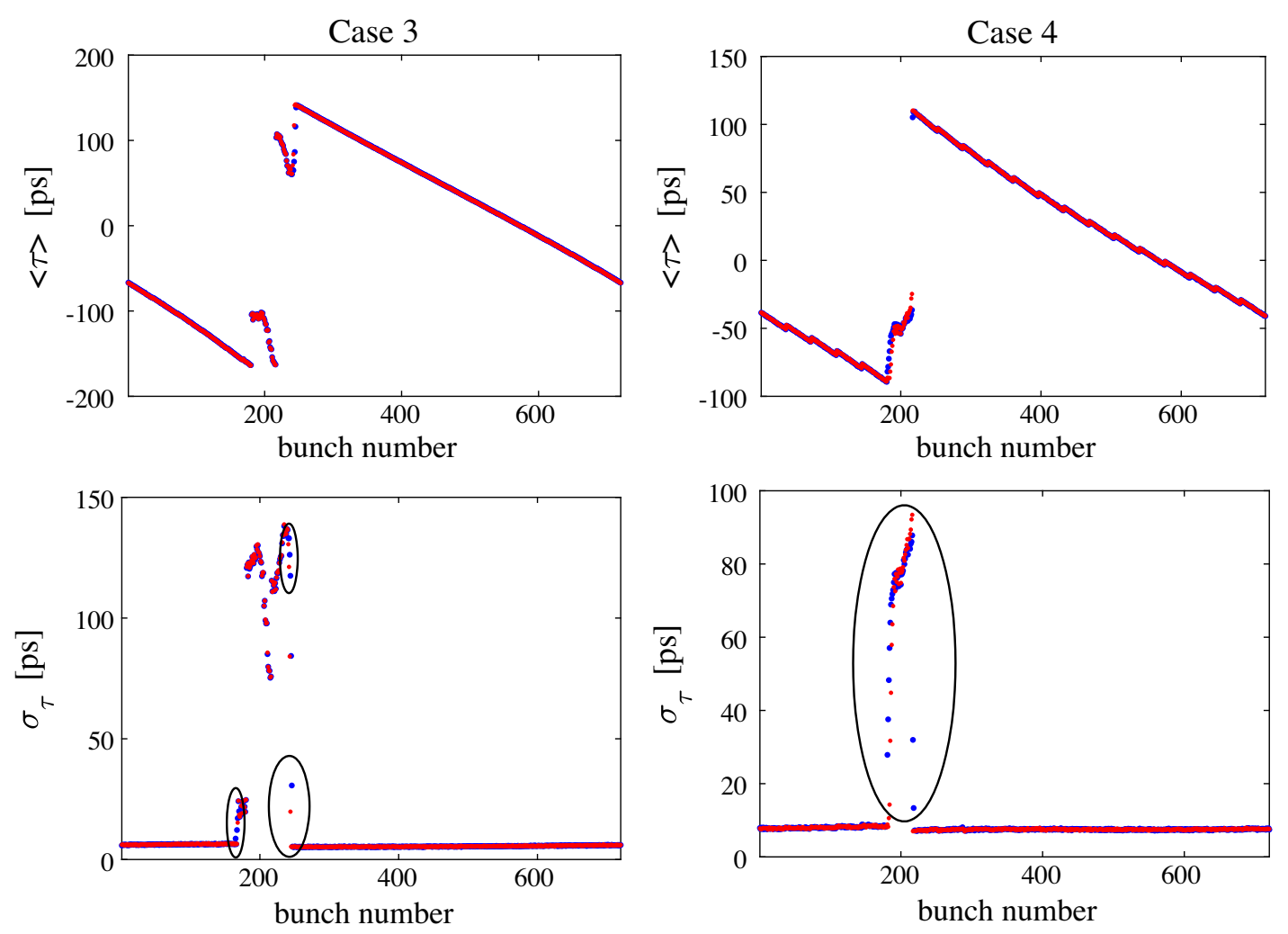

FIG. 10. Centroid positions (top) and rms lengths (bottom) vs bunch number for cases 3 (left) and 4 (right), given by STABLE at the 1 millionth (blue dots) and 10 millionth (red dots) tracking turns. The bunches that are moving during the whole tracking process are surrounded by an ellipse.

lengths with the bunch number, which are obtained by STABLE at 1 and 10 millionth tracking turns. According to the variation of rms lengths at this two tracking turns, it is easily found that most bunches eventually reach equilibrium states, while the rest of the bunches are moving all the time. These moving bunches are signed by an ellipse surrounding them in Fig. 10. It should be noted that the tracking results are not convergent and different moving bunches will be observed if we carry out another tracking simulation with a different number of macroparticles per bunch. However, the resulting centroid transients are generally close. It also should be noted that the semianalytical approach failed to reach convergence for both cases 3 and 4, so its results are not shown.

Ignoring the convergence, it is important to discuss the centroid transient and bunch lengthening. For case 3, we can see that the centroid positions vary in the range of -160 to $140 \mathrm{ps}$, which presents a much more severe centroid transient in comparison with case 1 . And some bunches have rms lengths more than $120 \mathrm{ps}$, which means that they are overstretched into two separated sub-bunches. However, most bunches have rms lengths around 6 ps. For case 4 with a higher MC voltage of $1.8 \mathrm{MV}$, the variation range of the centroid position is reduced to -90 to $110 \mathrm{ps}$, and the rms lengths of most bunches are increased to $8 \mathrm{ps}$. It indicates that, for the normal conducting case with $R / Q$ of $200 \Omega$, increasing the MC voltage can only improve the bunch lengthening to a limited extent. This can be explained by the fact that due to the large value of $R / Q$, the beam loading is still relatively strong even though the MC voltage is increased to $1.8 \mathrm{MV}$. Maybe using a high enough MC voltage can make it back to normal (as the case 2 shown in Fig. 9), but increasing the MC voltage will also reduce the natural bunch length, and at the same time will increase the rf power demands significantly. In turn, more HHCs may be needed, resulting in a larger total value of $R / Q$.

As for the bunch lengthening of the HALF storage ring, it can be clearly seen, from Figs. 9 and 10, that the passive normal conducting HHCs with a total $R / Q$ of $\sim 200 \Omega$ can cause severe centroid transient effects, dramatically degrade the average bunch lengthening, and thus is not suitable for HALF. If the Super-3HC with $R / Q$ of $90 \Omega$ is chosen for HALF, the present MC voltage is not enough and needs to be increased. Recall that we got good results with $R / Q$ of $45 \Omega$ in Sec. VII, therefore, if the MC voltage remains the same, a lower $R / Q$ is necessary.

\section{CONCLUSIONS}

In this paper, we first described a novel scheme to deal with the interaction of beam with resonant modes of $\mathrm{rf}$ cavities, where a new GPU-accelerated algorithm was 
proposed for the long-range interbunch interaction and a bin-wake convolution method was used for the short-range intrabunch interaction. This scheme can significantly enhance the parallelism of operations and thus the computational efficiency. A code based on MATLAB was developed to implement this scheme, which was named as STABLE. At present, the STABLE code can be used for studying longitudinal beam dynamics of arbitrary bunch trains, with the inclusion of short-range wake, beam loading of MC and HHC and a simple feedback model for beam loading compensation of MC. In the future, it will be extended to include arbitrary resonators so as to have the capability to evaluate the effects of HOMs in rf cavities.

Efficiency tests were carried out for STABLE on a desktop personal computer, which has a consumer grade GPU card with only 896 CUDA cores. The ALS-U with 284 bunches and HALF with 720 bunches were taken as two test examples. It was found that, with 1000 to 5000 macroparticles per bunch used in STABLE, the calculation time was comparable to or shorter than those of semianalytical methods. If a better GPU card with more CUDA cores is used, the computational efficiency can be further enhanced.

The STABLE code was benchmarked against a semianalytical method for an equilibrium case and the ELEGANT code for a nonequilibrium case. For the equilibrium case, the results were in good agreement. For the nonequilibrium case, both codes gave well a regular and periodic nonequilibrium state.

The STABLE code was first applied to studying the overstretching case for ALS-U with distributed gaps fill pattern. The tracking results revealed a periodic transient effect. We then applied STABLE to calculating the bunch lengthening for HALF with uniformly distributed gaps fill pattern. In the HALF case, superconducting and normal conducting cavities were considered. The tracking results showed that, in order to achieve good bunch lengthening, the passive normal conducting HHCs with $R / Q$ of $\sim 200 \Omega$ were not suitable for HALF and a superconducting HHC with lower $R / Q$ might be preferred.

Note that the fill pattern with distributed gaps was proposed to suppress the transient beam loading effect. However, through the tracking simulation, we found that, with a high $R / Q$, it was still very likely for the beam bunch to suffer from severe transient effect, which can significantly degrade the bunch lengthening. Therefore, an important conclusion can be drawn that a sufficiently low $R / Q$ of HHC is necessary to ensure good bunch lengthening for the HALF storage ring as well as other similar rings.

\section{ACKNOWLEDGMENTS}

We would like to thank the colleagues Fangfang $\mathrm{Wu}$ and Jian Pang at NSRL for helpful discussions, Tianyu Zhou for reinstalling the Linux system in the workstation, and Gangwen Liu for the helpful instruction for installing the parallel version of ELEGANT. This work was supported by National Natural Science Foundation of China (12105284) and the Fundamental Research Funds for the Central Universities (Grant No. WK2310000090).

\section{APPENDIX A: EQUIVALENT VOLTAGE PHASOR}

It is known that the voltage phasor produced by a resonant wake is evolved with a time factor of $(j-1 / 2 Q) \omega_{r}$. This characteristic will be used to derive the equivalent voltage phasor in the time domain. Given a charged bunch with a collection of macroparticles, where each macroparticle has a charge of $q, \tau=0$ is defined as the reference position related to the reference phase, and considering two particles, one is in the head and has $\tau_{j}<0$, the other is in the tail and has $\tau_{i}<0$. Then for the head particle, its equivalent voltage phasor at $\tau=0$ has the form

$$
\frac{q \omega_{r} R}{Q} e^{\left(j-\frac{1}{2 Q}\right) \omega_{r}\left(0-\tau_{j}\right)}=\frac{q \omega_{r} R}{Q} e^{\left(-j+\frac{1}{2 Q}\right) \omega_{r} \tau_{j}} .
$$

For the tail particle, its equivalent voltage phasor at $\tau=0$ can be given by

$$
\frac{q \omega_{r} R}{Q} e^{\left(j-\frac{1}{2 Q}\right) \omega_{r}\left(0-\tau_{i}\right)}=\frac{q \omega_{r} R}{Q} e^{\left(-j+\frac{1}{2 Q}\right) \omega_{r} \tau_{i}} .
$$

We can see that no matter the particle is in the head or the tail, their equivalent voltage phasors have the same form. Therefore, for a bunch composed of $\mathrm{N}$ macroparticles, the total equivalent voltage phasor at $\tau=0$ takes the form

$$
\frac{q \omega_{r} R}{Q} \sum_{k=1}^{N} \exp \left[\left(-j+\frac{1}{2 Q}\right) \omega_{r} \tau_{k}\right] .
$$

Note that the so-called equivalent voltage phasor is proposed just for the trailing bunches.

\section{APPENDIX B: VOLTAGE PHASOR OF RESONANT MODE WITH MODERATE Q FACTOR}

The resonant wake without any approximation is given by

$W(\tau)=\frac{\omega_{r} R}{Q} e^{-\frac{\omega_{r} \tau}{2 Q}}\left[\cos \left(\omega_{n} \tau\right)-\frac{\omega_{r}}{2 Q \omega_{n}} \sin \left(\omega_{n} \tau\right)\right] H(\tau)$,

where $H(\tau)$ is the Heaviside step function, and $\omega_{n}$ is

$$
\omega_{n}=\omega_{r} \frac{\sqrt{4 Q^{2}-1}}{2 Q},
$$

Eq. (B1) can also be given using a complex phasor 


$$
\begin{aligned}
W(\tau)= & \operatorname{Re}\left[\frac{\omega_{r} R}{Q} \exp \left(-\frac{\omega_{r} \tau}{2 Q}+j \omega_{n} \tau\right)\right] \\
& -\frac{\omega_{r}}{2 Q \omega_{n}} \operatorname{Im}\left[\frac{\omega_{r} R}{Q} \exp \left(-\frac{\omega_{r} \tau}{2 Q}+j \omega_{n} \tau\right)\right],
\end{aligned}
$$

where $\operatorname{Re}[]$ means to take the real part and $\operatorname{Im}[]$ means to take the imaginary part. If we let

$$
\tilde{V}(\tau)=\frac{\omega_{r} R}{Q} \exp \left(-\frac{\omega_{r} \tau}{2 Q}+j \omega_{n} \tau\right)
$$

then

$$
W(\tau)=\operatorname{Re}[\tilde{V}(\tau)]-\frac{\omega_{r}}{2 Q \omega_{n}} \operatorname{Im}[\tilde{V}(\tau)] .
$$

It means that we only need to record the complex phasor $\tilde{V}(\tau)$, which can be obtained by aforementioned Eqs. (3)-(5) and (8) where the phase factor of rotating and decaying should be replaced by

$$
j \frac{\sqrt{4 Q^{2}-1}}{2 Q}-\frac{1}{2 Q}
$$

and the initial $\tilde{V}_{c, 0}$ can be set to zero.

\section{APPENDIX C: THE CHOICE OF Q FACTOR FOR TRACKING}

For the case of HALF with a passive superconducting third-harmonic cavity, as the cavity has very large $Q\left(>1 \times 10^{8}\right)$, it requires very many tracking turns to make the results convergent and reliable, so it won't be used directly in tracking. A reduction of $Q$ will thus be used to reduce the necessary tracking turns, since it will only
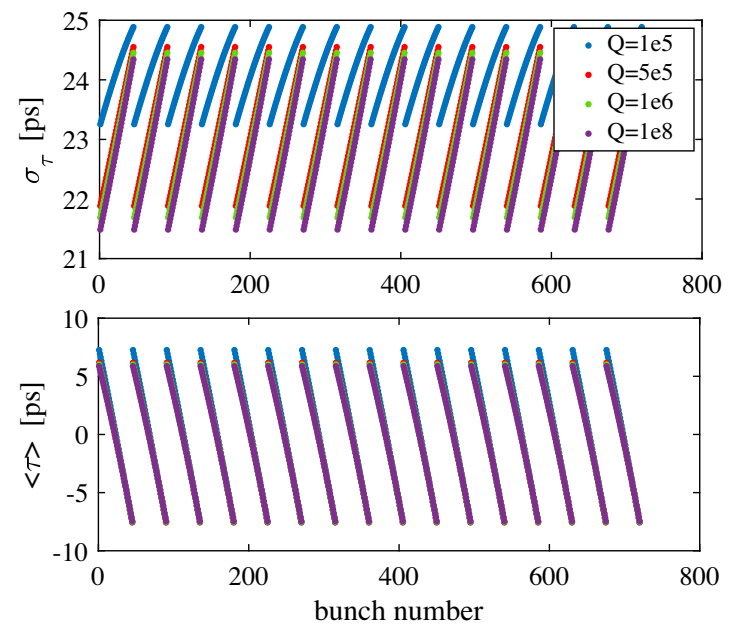

FIG. 11. RMS bunch length (top) and centroid position (bottom) vs bunch number for HALF with 720 bunches in filling case of uniformly distributed gaps, obtained by the semianalytical method for four kinds of $Q$ factor, $R / Q=45 \Omega$. Fill pattern: $16 \times(45 b+5 e)$. slightly change the tracking result when keeping the $R / Q$ unchanged by the reduction of the shunt impedance. Then it brings a question that how much $Q$ can be used and it will not affect the results too much.

Figure 11 shows the results of 4 kinds of $Q$, calculated by the semianalytical method, in the case of uniformly distributed gaps with 16 subtrains and 5 empty buckets between two adjacent subtrains. We can see that the results of $Q=5 \times 10^{5}$ are close enough to those of $Q=1 \times 10^{8}$. Therefore, for the superconducting cases discussed in Sec. VIII B, $Q=5 \times 10^{5}$ will be used to do the tracking.

[1] A. W. Chao, Physics of Collective Beam Instabilities in High Energy Accelerators (John Wiley \& Sons, New York, 1993).

[2] K. Y. Ng, Physics of Intensity Dependent Beam Instabilities (World Scientific, Singapore, 2006).

[3] T. Olsson, F. J. Cullinan, and A. Andersson, Self-consistent calculation of transient beam loading in electron storage rings with passive harmonic cavity, Phys. Rev. Accel. Beams 21, 120701 (2018).

[4] R. Warnock and M. Venturini, Equilibrium of an arbitrary bunch train in presence of a passive harmonic cavity: Solution through coupled Haïssinski equations, Phys. Rev. Accel. Beams 23, 064403 (2020).

[5] T. He, W. Li, Z. Bai, and L. Wang, Longitudinal equilibrium density distribution of arbitrary filled bunches in presence of a passive harmonic cavity and the short-range wakefield, Phys. Rev. Accel. Beams 24, 044401 (2021).

[6] R. Warnock, Equilibrium of an arbitrary bunch train in the presence of multiple resonator wakefields, Phys. Rev. Accel. Beams 24, 024401 (2021).

[7] F. J. Cullinan, Å. Andersson, and P. F. Tavares, Harmonic cavity stabilization of longitudinal coupled-bunch instabilities with a nonuniform fill, Phys. Rev. Accel. Beams 23, 074402 (2020).

[8] M. Ruprecht, Calculation of coupled bunch effects in the synchrotron light source BESSY VSR, Ph.D. thesis, Humboldt-Universität, Berlin, Germany, 2016.

[9] G. Skripka, R. Nagaoka, M. Klein, F. Cullinan, and P. F. Tavares, Simultaneous computation of intrabunch and interbunch collective beam motions in storage rings, Nucl. Instrum. Methods Phys. Res., Sect. A 806, 221 (2016).

[10] User's manual for ELEGANT, http://ops.aps.anl.gov/ manuals/elegant_latest/elegant.html.

[11] M. Migliorati and L. Palumbo, Multibunch and multiparticle simulation code with an alternative approach to wakefield effects, Phys. Rev. ST Accel. Beams 18, 031001 (2015).

[12] P. Schönfeldt, M. Brosi, M. Schwarz, J. L. Steinmann, and A.-S. Müller, Parallelized Vlasov-Fokker-Planck solver for desktop personal computers, Phys. Rev. Accel. Beams 20, 030704 (2017).

[13] H. Xu, U. Locans, A. Adelmann, and L. Stingelin, Calculation of longitudinal collective instabilities with mbtrack-cuda, Nucl. Instrum. Methods Phys. Res., Sect. A 922, 345 (2019). 
[14] K. Amyx, J. R. King, I. V. Pogorelov, M. Borland, and R. Soliday, Current statues of the GPU-accelerated ELEGANT, in Proceedings of IPAC 2014, Dresden, Germany (JACoW, Dresden, Germany, 2014), pp. 454-456, MOPME035, https://doi.org/10.18429/JACoW-IPAC2014-MOPME035.

[15] J. M. Byrd and M. Georgsson, Lifetime increase using passive harmonic cavities in synchrotron light sources, Phys. Rev. ST Accel. Beams 4, 030701 (2001).

[16] M. Pedrozzi, J.-Y. Raguin, W. Gloor, A. Anghel, M. Svandrlik, G. Penco, P.Craievich, A. Fabris, C. Pasotti,
E. Chiaveri, R. Losito, S. Marque, O. Aberle, P. Marchand, P. Bosland, S. Chel, P. Brédy, and G. Devanz, SLS operational performance with third harmonic superconducting system, in Proceedings of the 11th Workshop on RF Superconductivity, Lübeck/Travemünder, Germany (JACoW, Lübeck, Germany, 2003), pp. 91-94, MOP25, https://accelconf.web.cern.ch/SRF2003/papers/mop25.pdf.

[17] G. Bassi and J. Tagger, Longitudinal beam dynamics with a higher-harmonic cavity for bunch lengthening, Int. J. Mod. Phys. A 34, 1942040 (2019). 